Research Paper

\title{
A clinical study on plasma biomarkers for deciding the use of adjuvant corticosteroid therapy in broncho- pulmonary dysplasia of premature infants
}

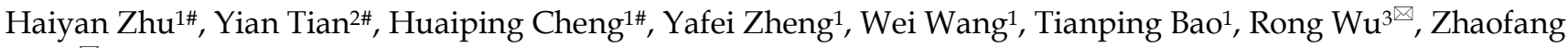 \\ $\operatorname{Tian}^{1 凶}$ \\ 1. Department of Neonatology, The Affiliated Huaian No.1 People's Hospital of Nanjing Medical University, Huai'an, Jiangsu, China. \\ 2. University of Barcelona, TPM-DTI, Barcelona, Catalunya, Spain \\ 3. Neonatal Medical Center, Huai'an Maternity and Child Healthcare Hospital, Yangzhou University Medical College, Huai' an, Jiangsu, China. \\ \#These authors contributed equally to this work as first author. \\ $\triangle$ Corresponding authors: Zhaofang Tian, The Affiliated Huaian No.1 People's Hospital of Nanjing Medical University, Huai'an, Jiangsu, 1 Huanghe Road \\ West, Huai'an, Jiangsu, 223300, China. E-mail: hayytzf@njmu.edu.cn; Tel: +8613905236862. Rong Wu, Neonatal Medical Center, Huai'an Maternity and Child \\ Healthcare Hospital, Yangzhou University Medical College, Huai'an, Jiangsu, E-mail:wr618@126.com; Tel:+86 18952386385.
}

(C) The author(s). This is an open access article distributed under the terms of the Creative Commons Attribution License (https://creativecommons.org/licenses/by/4.0/). See http://ivyspring.com/terms for full terms and conditions.

Received: 2021.01.25; Accepted: 2021.04.22; Published: 2021.04.29

\begin{abstract}
Objective: The study was designed to investigate some plasma markers which help us to decide the use of adjuvant corticosteroid therapy in bronchopulmonary dysplasia (BPD) of premature infants.

Methods: Thirty BPD infants were treated by dexamethasone. Among these cases, dexamethasone was significant effective in 10 cases, and no significant effective in 20 cases. These patients were divided into two groups as the significant effect (SE) group $(n=10)$ and the non-significant effect $(N E)$ group $(n=20)$ according to the curative effect of dexamethasone. Fifteen non-BPD infants with gestational age and gender matching were selected as the control group. Plasma samples before and after dexamethasone treatment were collected from three infants chosen randomly from SEG for the data-independent acquisition (DIA) analysis. ELISA was further used to detect the levels of differential proteins LRPI and S100A8 in all individuals, including SE, NE and control groups.

Results: DIA analysis results showed that after dexamethasone treatment, there were a total of 52 plasma proteins that showed significant differences, of which 43 proteins were down-regulated and 9 proteins were up-regulated. LRPI and S100A8 were two plasma proteins that were significantly changed after dexamethasone treatment. Compared with the control group, plasma LRPI was significantly increased in BPD. Interestingly, the plasma concentration of LRPI in the NE group was significantly higher than that in the SE group. S100A8, as an indicator of plasma inflammation, was significantly higher in BPD than the control group. Unlike LRPI, there was no significantly difference between the $S E$ and NE group $(P=0.279)$ before dexamethasone treatment.

Conclusion: Elevated plasma LRPI and S100A8 in BPD infants are two indicators that correlated with the efficacy of dexamethasone, and might be used as biomarkers for deciding the use of adjuvant corticosteroids therapy in the BPD.
\end{abstract}

Key words: bronchopulmonary dysplasia; proteome; biomarker; corticosteroids

\section{Introduction}

Bronchopulmonary dysplasia (BPD) is the result of a complex process in which several prenatal and/ or postnatal factors interfere with lower respiratory tract development, leading to a severe, lifelong disease. In the underlined disease the lungs and bronchi are damaged which destroys tissues in the alveoli of the lungs. Although its definition, epidemiology, pathophysiology, prevention, and management have continued to evolve. The prematurity of newborns is the major risk factor for BPD. However, the severity of the BPD differs from infant to infant [1]. Each year, over 10000 new BPD cases are reported in the US [2]. In China, the incidence of BPD in ultra-premature or ultra-low birth 
weight infants (ELBW) varied in $12.5 \%-48.1 \%[3,4]$.

Many non-pharmacologic and pharmacologic therapies have been suggested for lowering the lung's injury and tend to enhance the recovery of patients [5] i.e., protective ventilation strategies, surfactant supplementation, optimal oxygen saturation goals, and the antenatal corticosteroids [6]. The presently used drug therapies for BPD treatment are as following, caffeine [7], bronchodilator [8], surfactant [9], diuretics [10], inositol [11], corticosteroids[12], viral immunization [13] and cardiac medications [14] etc. However, these treatments are partially effective; the majority of the interventions tried so far have not proven to be beneficial in rigorous meta-analyses of eligible studies. And still, there is a lack of significant therapy for curing the underlined disease.

In BPD the corticosteroids are used to reduce the inflammatory processes that lead to the pathogenesis of the disease. Corticosteroids have a highly potent inhibitory activity against inflammation and have an important contribution to the prevention of BPD. With the risks for Perforated Bowel, hypertrophic cardiomyopathy, major neurosensory disability, and cerebral palsy (CP), the "early" dexamethasone therapy has not been recommended, and the "later" dexamethasone therapy can be used selectively [15]. The statement policy of an American Academy of Pediatrics was published in 2010 regarding the use of postnatal corticosteroids and admitted that the available data were not enough to support the corticosteroids recommendations [16]. Since the BPD infants have different therapeutic response to dexamethasone therapy, so it has an important clinical value to find biomarkers that can evaluate the effectiveness of the BPD affected infants for dexamethasone therapy.

DIA is a non-standard quantitative technique employed for the identification of differentially expressed proteins. This technique is well suited for the evaluation of lung injury in children to identify key biomarkers [17]. The purpose of the study is to find differentially expressed plasma proteins of BPD infants responsive to dexamethasone therapy by DIA technique to promote effective treatment of BPD infants.

\section{Materials and Methods}

\section{Patients}

The existing work was conducted in the Neonatal Intensive Care Unit of The Affiliated Huaian No.1 People's Hospital of Nanjing Medical University. At 36 weeks of postmenstrual age from Jan 2019 to Mar 2020, premature babies with gestational period $<32$ weeks were detected with
BPD. The diagnosed was based on "New consensus on BPD definitions and diagnostic criteria, developed in 2018 NICHD Seminar" (abbreviated as 2018 NICHD) [18]. The following inclusion criterion is applicable in the subjects: clinical diagnosis as BPD grade III, the details were as follows. Treatment with more than $21 \%$ oxygen for minimum of 28 days, required for $30 \%$ oxygen and/or positive pressure or discharge from the hospital, whichever comes first. Any one of the following exclusion criteria was applicable in the current study: I) There existed some congenital malformations such as digestive tract, respiratory tract, central nervous system, and heart; II) There existed ventilator-associated pneumonia (VAP); III) any glucocorticoid e.g., dexamethasone, hydrocortisone, budesonide, etc. administrated before the research. IV) Clinical signs of infection were existed. Totally, thirty BPD infants were enrolled in this study.

All eligible cases were treated with a 10-day dexamethasone course after diagnosing with BPD. The details are as follows: Infants received a 10-day tapering course of dexamethasone sodium phosphate $(0.15 \mathrm{mg} / \mathrm{kg}$ per day for 3 days, $0.10 \mathrm{mg} / \mathrm{kg}$ per day for 3 days, $0.05 \mathrm{mg} / \mathrm{kg}$ per day for 2days, and $0.02 \mathrm{mg} / \mathrm{kg}$ per day for 2 days; total of $0.89 \mathrm{mg} / \mathrm{kg}$ over 10 days [19]. Plasma samples were retained before and after dexamethasone therapy, respectively. The curative effect was judged immediately after 10-day course of dexamethasone. According to the curative effect of dexamethasone, these BPD infants were divided into two groups as the significant effect $(\mathrm{SE})$ group $(\mathrm{n}=10)$ and the non-significant effect $(\mathrm{NE})$ group $(n=20)$. SE was defined as patients with no need of respiratory support and oxygen dependence. $\mathrm{NE}$ was defined as lowering the inhaled oxygen concentration and/or the decrease in positive pressure support, or no change in inhaled oxygen concentration and/or no improvement in respiratory support. In addition, 15 non-BPD infants with gestational age and gender matching were selected as the control group. The approval for this clinical research was provided by the ethics committee of The Affiliated Huaian No.1 People's Hospital of Nanjing Medical University, and the relevant informed consent was signed from the parents before the trial. The registration number of the Chinese Clinical Trail Registry is ChiCTR2000033796 (http://www. chictr.org.cn/historyversionpub.aspx?regno $=$ ChiCTR 200003796).

\section{DA analysis}

Plasma samples before and after dexamethasone treatment were collected from three infants chosen randomly from SEG for the data-independent 
acquisition (DIA) analysis (Guangzhou Jidio Biotechnology Co. Ltd, Guangzou, China). Plasma samples were transferred into lysis buffer, and the proteins in lysates were concentrated, followed by proteins digesting via sequence-grade modified trypsin using the method of BCA Protein Assay Kit (Guangzhou Jidio Biotechnology Co. Ltd, Guangzhou, China). The digested peptides were dried to prepare for the analysis of nano-HPLC-MS/ MS. Unprocessed data obtained from DIA were analyzed and sort out via Spectronaut X (Biognosys AG, Switzerland) with default parameters. After Student's t-test, various expressed proteins (having $Q$ value $<0.05$ and Absolute AVG $\log 2$ ratio $>0.58$ ) were filtered.

\section{Enzyme-linked immunosorbet assay (ELISA)}

To confirm the differentially expressed proteins identified by DIA, we focused on LDL receptorrelated protein 1 (LRP1) and S100 calcium-binding protein A8 (S100A8). Venous blood was centrifuged at $1,000 \times \mathrm{g}$ to obtain a supernatant. The levels of LRP1 and S100A8 in the supernatant were identified via the ELISA kit (abcam, Cambridge, England) for the control group and the BPD group (including pre-and post- dexamethasone administration), following the given instructions of the manufacturer.

\section{Statistical analysis}

GraphPad Prism 8.0 (GraphPad Software Inc.) was used for statistical processing. The difference was evaluated by paired Student $t$ test (2-group comparisons) or ANOVA followed by the post hoc Tukey's Multiple Comparison Test (multi-group comparisons) as appropriate. Data are presented as the mean \pm SD. $\mathrm{P}<0.05$ was considered statistically significant.

\section{Results}

\section{The characteristics of BPD infants}

A total of 30 eligible infants were included in this study, of which 10 cases showed significant effect (SE), while 20 non-significant effects (NE) after dexamethasone treatment. The two groups' patients had similar perinatal characteristics, as depicted in Table 1. The difference was that three patients in the NE group had ventilator associated pneumonia, while none in the SE group. Three BPD patients were randomly selected from the SE group. The perinatal characteristics were presented in Table 2. The perinatal characteristics of the selected three BPD patients for DIA analyses were representative.
Table 1. Sociodemographic and prerandomization characteristics of study infants [mean \pm SD or $n(\%)$ ]

\begin{tabular}{|c|c|c|c|c|}
\hline Perinatal characteristics & $\begin{array}{l}\text { SE group } \\
(\mathrm{n}=10)\end{array}$ & $\begin{array}{l}\text { NE group } \\
(n=20)\end{array}$ & $\mathrm{X} 2 / \mathrm{t}$ & $\begin{array}{l}P \\
\text { value }\end{array}$ \\
\hline Gestational age (weeks) & $31.2 \pm 1.1$ & $31.5 \pm 1.4$ & 0.092 & 0.761 \\
\hline Birthweight (grams) & $1325.0 \pm 249.0$ & $1365.0 \pm 262.0$ & 0.053 & 0.816 \\
\hline Male sex [n (\%)] & $6(60)$ & $13(65)$ & 0.601 & 0.421 \\
\hline $\begin{array}{l}\text { Maternal dexamethasone } \\
\text { [n (\%)] }\end{array}$ & $9(90)$ & $18(90)$ & - & $>0.05$ \\
\hline Vaginal delivery [n (\%)] & $6(60)$ & $11(55)$ & 0.093 & 0.758 \\
\hline Apgar score at $5 \mathrm{~min}$ & $7 \pm 2$ & $7 \pm 2$ & - & $>0.05$ \\
\hline $\begin{array}{l}\text { Surfactant doses } \\
\text { administered [n (\%)] }\end{array}$ & $10(100)$ & $20(100)$ & - & $>0.05$ \\
\hline BPD grade (III) [n (\%)] & $10(100)$ & $20(100)$ & - & $>0.05$ \\
\hline $\begin{array}{l}\text { Respiratory distress } \\
\text { syndrome }[\mathrm{n}(\%)]\end{array}$ & $10(100)$ & $20(100)$ & - & $>0.05$ \\
\hline $\begin{array}{l}\text { Pulmonary hypertension } \\
\text { (PH) }[\mathrm{n}(\%)]\end{array}$ & $2(20)$ & $5(25)$ & 0.144 & 0.739 \\
\hline $\begin{array}{l}\text { Patent ductus arteriosus } \\
\text { (PDA) }[\mathrm{n}(\%)]\end{array}$ & $8(80)$ & $17(85)$ & 0.629 & 0.416 \\
\hline $\begin{array}{l}\text { Ventilator associated } \\
\text { pneumonia }[\mathrm{n}(\%)]\end{array}$ & $0(0)$ & $3(15)$ & - & $<0.01$ \\
\hline $\begin{array}{l}\text { Atrial septal defect (ASD) } \\
{[\mathrm{n}(\%)]}\end{array}$ & $8(80)$ & $18(90)$ & 0.838 & 0.341 \\
\hline $\begin{array}{l}\text { Ventricle septal defect (VSD) } \\
\text { [n (\%)] }\end{array}$ & $8(80)$ & $17(85)$ & 1.876 & 0.170 \\
\hline
\end{tabular}

Table 2. Sociodemographic and prerandomization characteristics of dexamethasone sensitive group infants $(n=10)$

\begin{tabular}{lll}
\hline Perinatal characteristics & DIA group & No-DIA group \\
\hline $\mathrm{n}$ & 3 & 7 \\
Gestational age, weeks & $30.0 \pm 1.0$ & $30.6 \pm 1.5$ \\
Birthweight, grams & $1350 \pm 160$ & $1365 \pm 180$ \\
Male sex, $\mathrm{n}(\%)$ & $2(66.7)$ & $4(57.1)$ \\
Maternal dexamethasone, $\mathrm{n}(\%)$ & $3(100.0)$ & $6(85.7)$ \\
Vaginal delivery, $\mathrm{n}(\%)$ & $2(66.7)$ & $4(57.1)$ \\
Apgar score at 5 min & $7 \pm 2$ & $7 \pm 2$ \\
Respiratory distress syndrome, $\mathrm{n}(\%)$ & $3(100.0)$ & $7(100.0)$ \\
Surfactant doses administered, $\mathrm{n}(\%)$ & $3(100.0)$ & $7(100.0)$ \\
BPD grade (III), $\mathrm{n}(\%)$ & $3(100.0)$ & $7(100.0)$ \\
\hline
\end{tabular}

There were no significant differences between groups. Data are mean \pm SD or $\mathrm{n}(\%)$.

\section{Differentially expressed proteins before and after dexamethasone administration in SE group}

There were 52 proteins displayed significant expression changes before and after dexamethasone treatment. In this cohort, the upregulation of 9 proteins and downregulation of 43 proteins were found, as indicated in Table 3. The volcanic map directly showed the location of various proteins between the groups that were compared, as depicted in Fig. 1. Hierarchical cluster analysis and Z-score homogenization were used to map the differentially expressed proteins in groups that were compared (each column represents a sample and each row represents a protein). The protein expression in different samples is expressed in different colors. The red color represents the elevated expression, while the blue color showed decreased expression level, as depicted in Fig. 2. 
Table 3. The information table of significant difference protein between after and before treatment of corticosteroids (top 10 proteins)

\begin{tabular}{llll}
\hline id & Symbol & $\log 2 \mathrm{fc}$ & $P$ value \\
\hline ENSG00000123384 & LRP1 & -2.764152705 & 0.006 \\
ENSG00000127083 & OMD & -1.713222868 & 0.013 \\
ENSG00000129538 & RNASE1 & -1.75694677 & 0.019 \\
ENSG00000143546 & S100A8 & -2.012564987 & 0.043 \\
ENSG00000011052 & NME & -13.43760929 & 0.0459 \\
ENSG00000167815 & PRDX2 & -1.75665693 & 0.165 \\
ENSG00000090013 & BLVRB & -2.106522502 & 0.183 \\
ENSG00000285043 & AC093512.2 & -2.21657055 & 0.211 \\
ENSG00000111341 & MGP & -2.787895194 & 0.222 \\
ENSG00000041982 & TNC & -2.69720062 & 0.366 \\
\hline
\end{tabular}

\section{Bioinformatics analyses of the differentially expressed proteins}

The analysis of GO (The Gene Ontology) indicated that the differentially expressed proteins participated in the cofactor metabolic process (GO : 0051186) and the catabolic process (GO : 0009056). The most significantly affected molecular functions were catalytic activity, binding, and structural activity relationship (Supplementary Online Fig. S1). Pathway analysis by KEGG suggested that the most significantly affected cascades were purine metabolism, pyrimidine metabolism, drug metabolism-other enzymes (Supplementary Online Fig. S2). We further map genes to each term of the DO database (http://disease-ontology.org/). The results of DO enrichment were shown in Supplementary Online Fig. S3. The DO items that pulmonary hypertension and persistent fetal circulation

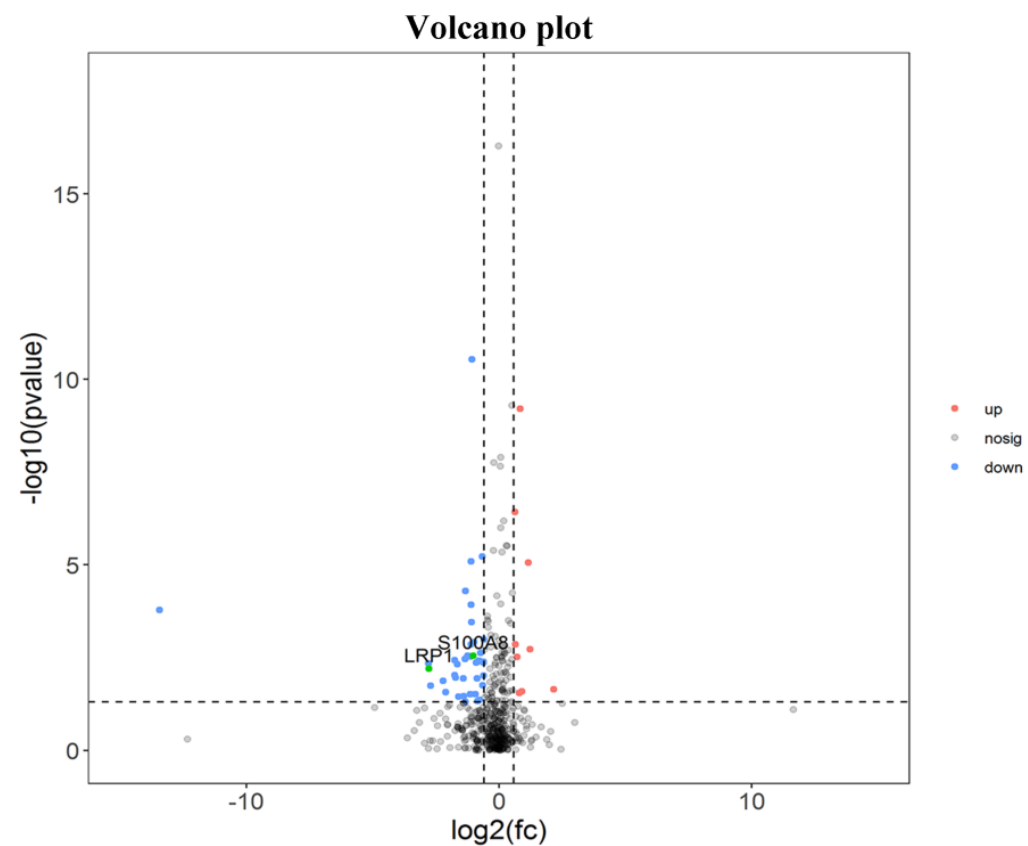

Figure 1. Distinct pulmonary proteomic patterns in T1 group and T2 group. Red represents up-regulated protein, blue represents down regulated protein, and black has no difference (T1 Pre-dexamethasone administration; T2 Post-dexamethasone administration). syndrome might relate to the pathogenesis of BPD. Then, we map the gene to each term of the react database (https://react.org/). The underlined results were depicted in Supplementary Online Fig. S4, and the top twelve Reactome items were significantly enriched in the gene $(p<0.05)$.

\section{Validation of changed LRPI and S100A8levels in the plasma samples}

ELISA analysis showed that LRP1 were significantly different among the three groups before dexamethasone administration $(\mathrm{F}=30.42, \mathrm{P}<0.001)$. Compared with the control group, plasma LRP1 was significantly increased in BPD. Interestingly, the plasma concentration of LRP1 in the NE group was significantly higher than that in the SE group (Fig. 3A). After dexamethasone administration than, the level of LRP1 were differentially downregulated either in the SE group or in the NE group (Fig. 3B). Likewise, S100A8 was significantly different among the three groups before dexamethasone administration $(\mathrm{F}=58.61, \mathrm{P}<0.001)$. The level of S100A8 was significantly higher in both the SE and NE group than that in the control group $(\mathrm{P}<0.05)$. However, there was no significantly difference between the $\mathrm{SE}$ and NE group $(\mathrm{P}=0.279)$ before dexamethasone treatment (Fig. 3C). The level of S100A8 was differentially downregulated in both the $\mathrm{SE}$ group and NE group after dexamethasone administration than that before dexamethasone administration. $(\mathrm{P}<0.05)$ (Fig. 3D).

\section{Discussion}

In this study, we used DIA technology to analyze the plasma proteins of BPD infants before and after dexamethasone treatment. We found that both LRP1 and S00A8 decreased significantly after dexamethasone treatment. If the baseline LRP1 is high, it suggests that dexamethasone may not show significant effectiveness. This provides a basis for clinical selection of adjuvant corticosteroids to treat BPD. 


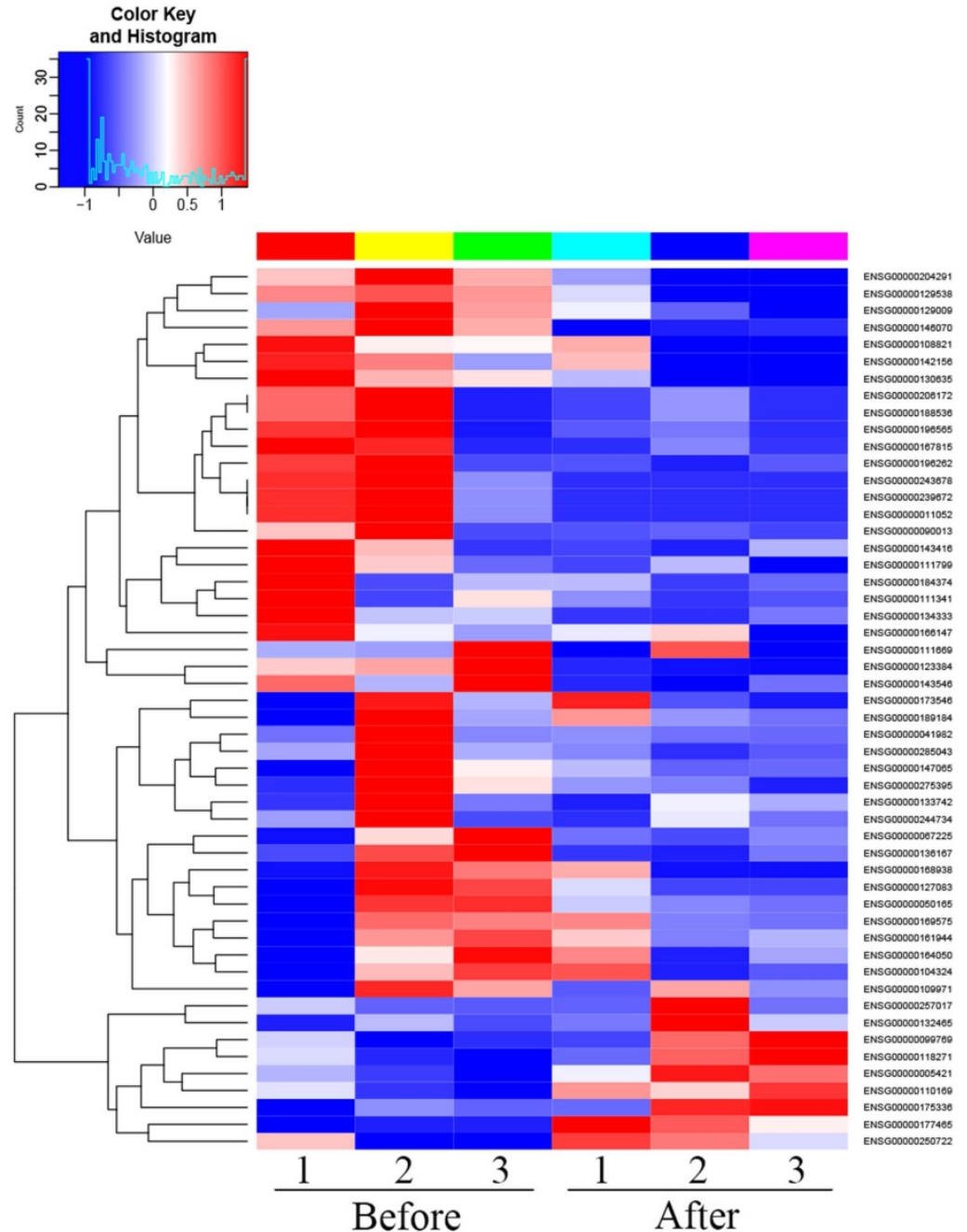

Figure 2. Heat map of differentially expressed proteins (T1 Pre- dexamethasone administration; T2 Pos-t dexamethasone administration).

A

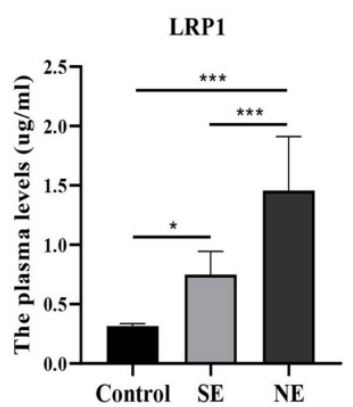

C

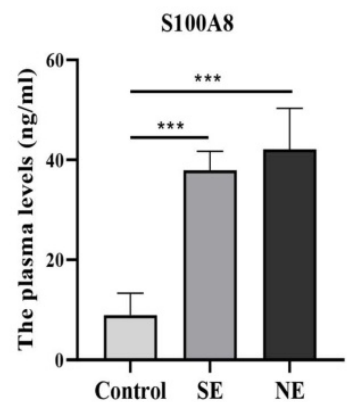

B

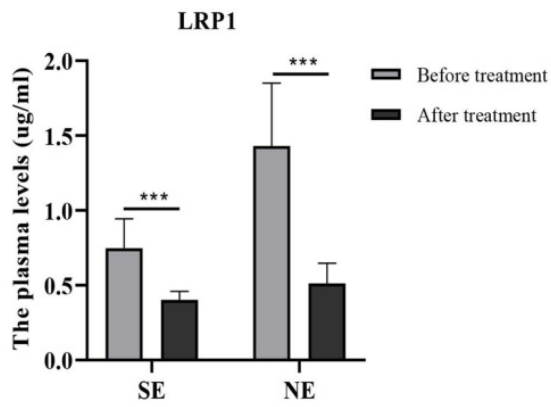

D

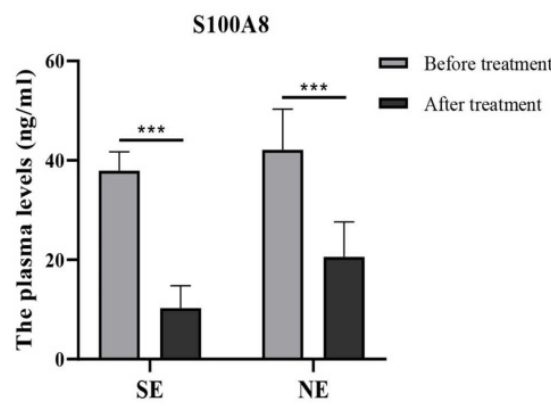

Figure 3. The levels of LRPI and S100A8 in the plasma of different groups. ${ }^{*} P<0.05, * * * P<0.001$ (SE-the significant effect group, NE-the non-significant effect group, control-non-BPD group). 
Dexamethasone had been widely used since the 1980s because of its potent activity against inflammation. The underlined corticosteroid can significantly change the course of BPD and decrease the BPD severity [20]. However, the adverse effects (such as cerebral palsy and major neurosensory disability, etc.) caused by glucocorticoids were gradually found from late 1990 [21].Therefore, in 2002, the American Academy of Pediatrics had suggested that the use of glucocorticoids in BPD should be more cautious [22]. Then the therapeutic use of corticosteroids in BPD had reduced effectively, but the rate of BPD had increased [23]. In recent years, glucocorticoid use in BPD infants has been increased. A European study revealed that $13.9 \%$ of premature infants (aged less than 30 weeks) received corticosteroid treatment after birth [24], however, in Japan the use of systemic steroid was published by $56 \%$ of units in $2005,57 \%$ in 2010 , and $68 \%$ of units in 2015 , respectively [25]. There is still no consensus on the use of corticosteroids in BPD [26, 27, 28]. In our study, 10 BPD infants were found to be significantly positive response to dexamethasone treatment, and the efficacy of the other 20 patients is not very satisfactory. The results suggested that some sensitive biomarkers are needed to choose the use of adjuvant corticosteroid therapy in BPD.

Protein mass spectrometry had been applied to some experimental studies regarding $\operatorname{BPD}[29,30]$, and clinical research by DIA indicated that calcium-associated proteins can be used as sensitive markers for BPD progress [31]. In this study, the results revealed that a total of 52 proteins were differentially expressed before and after dexamethasone treatment. In these differentially expressed proteins, the decreased expression of 43 proteins and elevated expression of 9 proteins were determined. The underlined differentially expressed proteins were further evaluated by literature search and the LRP1 and S100A8 were found to be closely correlated with lung diseases and/or glucocorticoids.

In this study, the plasma LRP1 level in the two BPD subgroups was significantly increased than that in the control group before dexamethasone administration. We also found that, the levels of LRP1 in the SE group were lower than those in the NE group before dexamethasone treatment. These results suggested that LRP1 might be either a diagnostic index or a target of dexamethasone therapy for BPD. These results suggest that a high baseline level of LRP1 may indicate that dexamethasone is not significant effective. This will provide a basis for clinical selection of dexamethasone to treat BPD.

LRP1 was first discovered in 1988, and its amino acid sequence was found to be similar to the LDL receptor. LRP1 is a cell surface receptor and regulates cellular and molecular mechanisms that drive the pathophysiological inflammatory reactions and reorganization of tissues in many organs [32]. In current decades, $\mathrm{LRP}_{1}$ has been evolved as a significant regulator of the inflammatory reactions. LRP1 negatively regulates adaptive immune responses in the site of HDM-induced eosinophilic airway inflammation [33]. LRP has a close association with dexamethasone. The expression of LRP was up-regulated by dexamethasone, and down-regulated by lipopolysaccharide (LPS), gamma interferon (IFN-gamma) or a combination of both. LRP was less sensitive to dexamethasone in activated astrocytes than in microglia [34]. Dexamethasone increases cell-surface LRP activity in HepG2 cells by increasing the steady state mRNA levels and suggest that post-transcriptional mechanisms play a role in controlling LRP mRNA levels [35].

Calprotectin S100A8 (S100 calcium binding protein A8, S100A8) is an important member of calcium binding protein family. The evaluation of its role in the pathogenesis of respiratory diseases or its usefulness as a biomarker for the appropriate diagnosis and prognosis of lung diseases have only gained attention in recent years [36]. S100A8/ A9 is an essential factor for neutrophil recruitment to lungs, and S100A8 promotes acute lung injury via Toll-like receptor 4-dependent activation of AECs [37]; S100A8/A9 serum levels along with chemokines are useful in distinguishing between active tuberculosis and asymptomatic Mycobacterium tuberculosisinfected latent individuals, and targeting S100A8/A9 pathways as host-directed therapy for TB [38]; Calprotectin is significantly elevated in the sputum of Bronchiolitis obliterans patients and reflects ongoing neutrophilic inflammation [39]; serum S100A8/ S100A9 was negatively associated with pulmonary function in acute exacerbation COPD(AE-COPD) patients, indicating that the serum S100A8/S100A9 heterodimer may be involved in the progression of $\mathrm{AE}-\mathrm{COPD}$, and may be a relevant serum biomarker in the diagnosis for AE-COPD [40]. Elevated S100A8/ S100A9 expression causes glucocorticoid resistance in MLL-rearranged infant acute lymphoblastic leukemia [41]. We found that S100A8 levels were significantly different between BPD group and the control group. Unlike LRP1, there was no difference in the level of S100A8 between the SE and NE groups.

In conclusion, we determined some differentially expressed plasma proteins of the dexamethasone significant effect infants with BPD via the DIA technique, among which, LRP1 and S100A8 might be the biomarkers for BPD diagnosis and deciding the use of adjuvant corticosteroids therapy in the BPD 
infants. This study is only a single center study and the sample number of is still small. In order to support the conclusions of this study, a large sample and multicenter study is necessary.

\section{Supplementary Material}

Supplementary figures.

http://www.medsci.org/v18p2581s1.pdf

\section{Acknowledgements}

\section{Author Contributions}

ZF T, HY Z and YA $T$ conceptualized and designed the study, extracted the data, analyzed the data, drafted the initial manuscript, and reviewed and revised the manuscript. R.W., HP.C., YF.Z., and W.W. conceptualized and designed the study, extracted the data, and reviewed and revised the manuscript. TP B designed the study, extracted the data, and reviewed and revised the manuscript. All authors approved the final manuscript as submitted).

\section{Funding}

This study was financially supported by Jiangsu maternal and child health science and technology project (F201909).

\section{Availability of data and materials}

The dataset generated or analyzed during this study can be made available to interested researchers by the authors of his article upon reasonable request.

\section{Statement of Ethics}

Ethical approval to conduct this study was obtained from by the ethics committee of The Affiliated Huaian No. 1 People's Hospital of Nanjing Medical University (Trial registration ID: ChiCTR2000033796). The guidelines followed the CONSORT 2010 Explanation and Elaboration. The statements of informed consent were signed by parents of the neonates; all the patent-related information was anonymized.

\section{Competing Interests}

The authors have declared that no competing interest exists.

\section{References}

1. Stoll BJ, Hansen NI, Bell EF, et al. Trends in Care Practices, Morbidity, and Mortality of Extremely Preterm Neonates, 1993-2012. JAMA. 2015; 314(10): 1039-1051.

2. Martin JA, Osterman MJK. Describing the Increase in Preterm Births in the United States, 2014-2016. NCHS Data Brief. 2018; 312:1-8.

3. Kong $\mathrm{X}, \mathrm{Xu} \mathrm{F}, \mathrm{Wu} \mathrm{R}$, et al. Neonatal mortality and morbidity among infants between 24 to 31 complete weeks: a multicenter survey in China from 2013 to 2014. BMC Pediatr. 2016; 16(1):174.

4. Lin HJ, Du LZ, Ma XL, et al. Mortality and Morbidity of Extremely Low Birth Weight Infants in the Mainland of China: A Multi-center Study. Chin Med J (Engl). 2015; 128(20):2743-50.
5. Jain D, Bancalari E. Bronchopulmonary dysplasia: clinical perspective. Birth Defects Res A Clin Mol Teratol. 2014; 100(3):134-144.

6. Michael Z, Spyropoulos F, Ghanta S,et al. Bronchopulmonary Dysplasia: An Update of Current Pharmacologic Therapies and New Approaches. Clin Med Insights Pediatr. 2018; 12:1179556518817322.

7. Schmidt B, Roberts RS, Davis P, et al. Caffeine therapy for apnea of prematurity. N Engl J Med. 2006; 354(20): 2112-2121.

8. Stewart A, Brion LP, Ambrosio-Perez I. Diuretics acting on the distal renal tubule for preterm infants with (or developing) chronic lung disease. Cochrane Database Syst Rev. 2011; 2011(9):CD001817.

9. Rigo V, Lefebvre C, Broux I. Surfactant instillation in spontaneously breathing preterm infants: a systematic review and meta-analysis. Eur J Pediatr. 2016; 175(12):1933-1942.

10. Ng G, da Silva O, Ohlsson A. Bronchodilators for the prevention and treatment of chronic lung disease in preterm infants. Cochrane Database Syst Rev. 2016; 12(12):CD003214.

11. Phelps DL,Ward RM,Williams RL, et al. Safety and pharmacokinetics of multiple dose myo-inositol in preterm infants. Pediatr Res. 2016;80(2):209-217.

12. Onland W, Offringa M, van Kaam A. Late ( $\geq 7$ days) inhalation corticosteroids to reduce bronchopulmonary dysplasia in preterm infants. Cochrane Database Syst Rev. 2017; 8(8):CD002311.

13. Darlow BA, Graham PJ, Rojas-Reyes MX. Vitamin A supplementation to prevent mortality and short- and long-term morbidity in very low birth weight infants. Cochrane Database Syst Rev. 2016; 2016(8):CD000501.

14. Guillot M, Offringa M, Lacaze-Masmonteil T, et al. Cell-based therapy for bronchopulmonary dysplasia in preterm infants 1. Can J Physiol Pharmacol. 2019; 97(3):232-234.

15. Doyle LW, Cheong JL, Ehrenkranz RA, et al. Early ( $<8$ days) systemic postnatal corticosteroids for prevention of bronchopulmonary dysplasia in preterm infants. Cochrane Database Syst Rev. 2017; 10(10):CD001146.

16. Watterberg KL. American Academy of Pediatrics. Committee on Fetus and Newborn. Policy statement postnatal corticosteroids to prevent or treat bronchopulmonary dysplasia. Pediatrics. 2010; 126(4):800-808.

17. Pereira-Fantini PM, Tingay DG. The proteomics of lung injury in childhood: challenges and opportunities. Clin Proteomics. 2016; 13:5.

18. Higgins RD, Jobe AH, Koso-Thomas M, et al. Bronchopulmonary Dysplasia: Executive Summary of a Workshop. J Pediatr. 2018; 197:300-308.

19. Lex W Doyle, Peter G Davis, Colin J Morley, et al. Low-dose dexamethasone facilitates extubation among chronically ventilator-dependent infants: a multicenter, international, randomized, controlled trial. Pediatrics. 2006; 117(1):75-83.

20. Mammel MC, Green TP, Johnson DE,et al. Controlled trial of dexamethasone therapy in infants with bronchopulmonary dysplasia. Lancet. 1983; 1(8338): 1356-1358.

21. O'Shea TM, Kothadia JM, Klinepeter KL, et al. Randomized placebo-controlled trial of a 42-day tapering course of dexamethasone to reduce the duration of ventilator dependency in very low birth weight infants: outcome of study participants at 1-year adjusted age. Pediatrics. 1999; 104(1 Pt 1):15-21.

22. Committee on Fetus and Newborn. Postnatal corticosteroids to treat or prevent chronic lung disease in preterm infants. Pediatrics. 2002; 109(2): 330-338

23. Yoder BA, Harrison M, Clark RH. Time-related changes in steroid use and bronchopulmonary dysplasia in preterm infants. Pediatrics. 2009; 124(2): 673-679.

24. Nuytten A, Behal $\mathrm{H}$, Duhamel A et al. Evidence-Based Neonatal Unit Practices and Determinants of Postnatal Corticosteroid-Use in Preterm Births below 30 Weeks GA in Europe. A Population-Based Cohort Study [published correction appears in PLoS One. 2017 Feb 13;12 (2):e0172408]. PLoS One. 2017; 12(1):e0170234.

25. Miyake F, Ito $\mathrm{M}$, Minami $\mathrm{H}$, et al. Management of bronchopulmonary dysplasia in Japan: A 10-year nationwide survey. Pediatr Neonatol. 2020; 61(3):

272-278

26. Bassler D, Shinwell ES, Hallman M, et al. Long-Term Effects of Inhaled Budesonide for Bronchopulmonary Dysplasia. N Engl J Med. 2018; 378(2): 148-157.

27. Onland W, Cools F, Kroon A, et al. Effect of Hydrocortisone Therapy Initiated 7 to 14 Days After Birth on Mortality or Bronchopulmonary Dysplasia Among Very Preterm Infants Receiving Mechanical Ventilation: A Randomized Clinical Trial. JAMA. 2019; 321(4):354-363

28. Baud O, Maury L, Lebail F, et al. Effect of early low-dose hydrocortisone on survival without bronchopulmonary dysplasia in extremely preterm infants (PREMILOC): a double-blind, placebo-controlled, multicentre, randomised trial. Lancet. 2016; 387(10030):1827-1836.

29. Lu X, Wang C, Wu D, et al. Quantitative proteomics reveals the mechanisms of hydrogen-conferred protection against hyperoxia-induced injury in type II alveolar epithelial cells. Exp Lung Res. 2018; 44(10):464-475.

30. Shrestha AK, Gopal VYN, Menon RT, Hagan JL, Huang S, Shivanna B. Lung omics signatures in a bronchopulmonary dysplasia and pulmonary hypertension-like murine model. Am J Physiol Lung Cell Mol Physiol. 2018; 315(5):L734-L741.

31. Magagnotti C, Matassa PG, Bachi A, et al. Calcium signaling-related proteins are associated with broncho-pulmonary dysplasia progression. J Proteomics. 2013; 94:401-412 
32. Wujak L, Schnieder J, Schaefer L,et al. LRP1: A chameleon receptor of lung inflammation and repair. Matrix Biol. 2018; 68-69:366-381.

33. Mishra A, Yao X, Saxena A, et al. Low-density lipoprotein receptor-related protein 1 attenuates house dust mite-induced eosinophilic airway inflammation by suppressing dendritic cell-mediated adaptive immune responses. J Allergy Clin Immunol. 2018; 142(4):1066-1079.e6.

34. Marzolo MP, von Bernhardi R, Bu G, et al. Expression of alpha(2)macroglobulin receptor/low density lipoprotein receptor-related protein (LRP) in rat microglial cells. J Neurosci Res. 2000; 60(3):401-411.

35. Kancha RK, Hussain MM. Up-regulation of the low density lipoprotein receptor-related protein by dexamethasone in HepG2 cells. Biochim Biophys Acta. 1996; 1301(3):213-220

36. Kotsiou OS, Papagiannis D, Papadopoulou R et al. Calprotectin in Lung Diseases. [J]. Int J Mol Sci. 2021; 22(4):1706.

37. Deblina Chakraborty ,Stefanie Zenker, Jan Rossaint, et al. Alarmin S100A8 Activates Alveolar Epithelial Cells in the Context of Acute Lung Injury in a TLR4-Dependent Manner. Front Immunol. 2017; 8:1493.

38. Ninecia R S, Rosemary VS, Noor AH, et al. S100A8/A9 regulates CD11b expression and neutrophil recruitment during chronic tuberculosis. J Clin Invest. 2020; 130(6):3098-3112.

39. S. P. Jerkic, F. Michel, H. Donath, et al. Calprotectin as a new sensitive marker of neutrophilic inflammation in patients with bronchiolitis obliterans. Mediat. Inflamm. 2020; 2020:1-7.

40. SJ Huang, ZN Ding, HX Xiang, et al. Association between Serum S100A8/ S100A9 Heterodimer and Pulmonary Function in Patients with Acute Exacerbation of Chronic Obstructive Pulmonary Disease. Lung. 2020: 198(4): 645-652.

41. Spiikers-Hagelstein JA, Schneider P, Hulleman E,et al. Elevated S100A8/ S100A9 expression causes glucocorticoid resistance in MLL-rearranged infant acute lymphoblastic leukemia. Leukemia. 2012; 26(6): 1255-1265. 